\title{
Ergodicity and partial hyperbolicity on the 3-torus
}

\author{
Andy Hammerlindl and Raúl Ures
}

September 29, 2018

\begin{abstract}
If a non-ergodic, partially hyperbolic diffeomorphism on the 3-torus is homotopic to an Anosov diffeomorphism $A$, it is topologically conjugate to $A$.
\end{abstract}

\section{Introduction}

Ergodicity is commonplace among partially hyperbolic diffeomorphisms, and so an interesting question is when such systems fail to be ergodic. We study this question in the specific case of the 3 -torus, proving the following.

Theorem 1.1. Suppose $f: \mathbb{T}^{3} \rightarrow \mathbb{T}^{3}$ is a conservative partially hyperbolic $C^{2}$ diffeomorphism homotopic to a linear Anosov map $A: \mathbb{T}^{3} \rightarrow \mathbb{T}^{3}$. If $f$ is not ergodic, it is topologically conjugate to $A$.

This research is motivated by the study of stable ergodicity. In the space of conservative $C^{2}$ diffeomorphisms, an element $f$ is stably ergodic if every nearby diffeomorphism is also ergodic. All Anosov diffeomorphisms are ergodic and they form an open subset of the space of diffeomorphisms. Therefore, every such system is an example of a stably ergodic diffeomorphism. In fact, for decades, these were the only known examples.

Then, Grayson, Pugh, and Shub studied the time-one map of the geodesic flow on a surface of constant negative curvature, showing it was also stably ergodic [6]. While this diffeomorphism has some hyperbolic behaviour, it acts as an isometry along the orbits of the flow. In particular, it is an example of a partially hyperbolic system, a diffeomorphism $f: M \rightarrow M$ with an invariant splitting of the tangent bundle $T M=E^{u} \oplus E^{c} \oplus E^{s}$, such that vectors in $E^{u}$ are exponentially expanded under iteration, those in $E^{s}$ are exponentially contracted, and where any expansion or contraction of the center bundle $E^{c}$ is weak in comparison. (The next section gives a precise definition.)

Further research yielded a wealth of partially hyperbolic examples of stable ergodicity, leading to the following conjecture of Pugh and Shub [18, [17. 
Conjecture 1.2. Stable ergodicity is open and dense in the space of conservative, partially hyperbolic $C^{2}$ diffeomorphisms.

This conjecture has been proved in a number of special cases, including [18] 13 10 4 3] 1. Recently, Avila, Crovisier, and Wilkinson announced a proof of a weaker version of the conjecture where they show density, but only in the $C^{1}$ topology. A key ingredient in the proofs of each of these results is a property called accessibility. A partially hyperbolic diffeomorphism is accessible if any two points $x, y \in M$ can be connected by a concatenation of paths, each path tangent either to $E^{u}$ or $E^{s}$.

Pugh and Shub split Conjecture 1.2 into two subconjectures.

Conjecture 1.3. Accessibility holds on an open and dense subset of the space of partially hyperbolic diffeomorphisms (conservative or not).

Conjecture 1.4. Every accessible conservative partially hyperbolic diffeomorphism is ergodic.

As with 1.2, these conjectures have been proven in many special cases. See 23] for a recent survey. In particular, the conjectures are true when the center bundle $E^{c}$ is one-dimensional.

Theorem 1.5 (Hertz-Hertz-Ures [10]). Accessibility is open and dense among conservative partially hyperbolic diffeomorphisms with one-dimensional center.

Theorem 1.6 (Hertz-Hertz-Ures [10], Burns-Wilkinson [4]). Every conservative, accessible, partially hyperbolic $C^{2}$ diffeomorphism with one-dimensional center is ergodic.

Thus, in this setting, the generic partially hyperbolic diffeomorphism is ergodic and an interesting question is to describe the non-ergodic ones. Even in the simplest case, where the manifold is three-dimensional and each of the bundles $E^{u}, E^{c}$, and $E^{s}$ is one-dimensional, this is a difficult open problem.

Question 1.7. Which 3-manifolds support non-ergodic partially hyperbolic diffeomorphisms? Further, what special properties do these non-ergodic systems have?

As a motivating example, first consider a hyperbolic toral automorphism $A: \mathbb{T}^{2} \rightarrow \mathbb{T}^{2}$ such as the map induced by the matrix $\left(\begin{array}{ll}2 & 1 \\ 1 & 1\end{array}\right)$. Define $f$ on $\mathbb{T}^{2} \times[0,1]$ as $A$ times the identity map on $[0,1]$. This is a non-ergodic partially hyperbolic diffeomorphism on a manifold with boundary. The stable $E^{s}$ and unstable $E^{u}$ directions come from the hyperbolic map $A$ and are tangent to the surfaces $\mathbb{T}^{2} \times\{t\}$. The center direction is tangent to fibers of the form $\{x\} \times[0,1]$.

To construct an example without boundary, suppose $B: \mathbb{T}^{2} \rightarrow \mathbb{T}^{2}$ is another toral automorphism that commutes with $A$. Then, the identification $(x, 1) \sim$ $(B x, 0)$ on $\mathbb{T}^{2} \times[0,1]$ produces a closed manifold $M_{B}$ with a non-ergodic partially hyperbolic diffeomorphism $f_{B}: M_{B} \rightarrow M_{B}$ coming from $f$.

If $B$ is the identity, $M_{B}$ is the 3 -torus. If $B$ is minus identity, that is, $B(x)=-x$ on $\mathbb{T}^{2}=\mathbb{R}^{2} / \mathbb{Z}^{2}$, then $M_{B}$ is double covered by the 3 -torus. The 
only remaining possibility is that $B$ is hyperbolic, in which case $f_{B}$ can be thought of as the time-one map of an Anosov flow on $M_{B}$.

While these simple constructions do not give all possible examples of nonergodic partially hyperbolic diffeomorphisms in dimension three, they do give all of the manifolds where such non-ergodic examples are known to exist. This fact and the results of [11] lead to the following conjectures.

Conjecture 1.8 (Hertz-Hertz-Ures [11). If a 3-manifold $M$ supports a nonergodic partially hyperbolic diffeomorphism, then $M=M_{B}$ (as defined above) where $B$ is \pm id or is hyperbolic.

Each example $f_{B}$ contains embedded tori tangent to $E^{u} \oplus E^{s}$. Such tori are clear obstructions to accessibility and they occur in every known non-ergodic example.

Conjecture 1.9 (Hertz-Hertz-Ures). For every non-ergodic partially hyperbolic diffeomorphism on a 3-manifold, $M$, there is an embedded, periodic, incompressible torus tangent to $E^{u} \oplus E^{s}$.

Such a torus is an example of an Anosov torus (as defined and studied in [14]) and its existence implies that $M$ must be one of the $M_{B}$ discussed above. In particular, if Conjecture 1.9 is true, then Conjecture 1.8 is also true as a consequence.

Suppose a diffeomorphism $f: M^{3} \rightarrow M^{3}$ contains a torus $S=f^{k}(S)$ as in Conjecture 1.9. Since $S$ is incompressible, $\pi_{1}(S)$ injects into $\pi_{1}(M)$ and so $\pi_{1}(M)$ contains a copy of $\mathbb{Z}^{2}$ invariant under the group automorphism $f_{*}^{k}$.

If no such subgroup exists, then no such torus exists. Unfortunately, for the manifolds under consideration, this technique to rule out tori only works in one specific case.

Proposition 1.10. Suppose $f$ is a diffeomorphism of $M_{B}$ where $B$ is \pm id or is hyperbolic. Then, exactly one of the following holds:

- $\pi_{1}\left(M_{B}\right)$ has an $f_{*}$-invariant subgroup isomorphic to $\mathbb{Z}^{2}$.

- $M_{B}=\mathbb{T}^{3}$ and $f_{*}$ is hyperbolic (when regarded as a $3 \times 3$ matrix).

The proof is basic group theory and is left as an exercise.

This proposition, taken with Conjecture 1.9, suggests the following conjecture, which was the main motivation in developing Theorem 1.1

Conjecture 1.11. Suppose $f: \mathbb{T}^{3} \rightarrow \mathbb{T}^{3}$ is a conservative partially hyperbolic $C^{2}$ diffeomorphism homotopic to a linear Anosov map $A: \mathbb{T}^{3} \rightarrow \mathbb{T}^{3}$. Then, $f$ is ergodic.

Theorem 1.1 instead shows that under these assumptions, a system is either ergodic, or is topologically conjugate to a well-understood, linear, ergodic example. This answers the question in spirit, but as the conjugacy to the Anosov system may not be absolutely continuous, a counter example is still possible. Such a counterexample must be highly pathological in nature. 
Suppose $f: \mathbb{T}^{3} \rightarrow \mathbb{T}^{3}$ is a conservative, non-ergodic, partially hyperbolic diffeomorphism homotopic to Anosov. Then:

- the conjugacy $h$ given by Theorem 1.1 satisfies $h\left(W_{f}^{*}\right)=W_{A}^{*}$ for $*=u, s, c$,

- the central Lyapunov exponent of $f$ is zero almost everywhere,

- $f$ fails to be 3-normally hyperbolic, that is, at some point $p \in \mathbb{T}^{3}$, the splitting fails to satisfy the inequality

$$
\left\|\left.T_{p} f\right|_{E^{s}}\right\|<\left\|\left.T_{p} f\right|_{E^{c}}\right\|^{3}<\left\|\left.T_{p} f\right|_{E^{u}}\right\| .
$$

Note that by the second condition, the center must be non-uniformly close to an isometry, but by the third condition, it cannot be uniformly close to an isometry. We give the precise statements and proofs of these three listed properties in Sections 6, 7, and 8 respectively.

Under addition assumptions, similar results hold for higher-dimension tori. These are described in Section 5 .

\section{Definitions}

Functions $f$ and $g$ are topologically conjugate if there is a homeomorphism $h$ such that $f \circ h=h \circ g$.

A diffeomorphism of a manifold is conservative if it preserves a finite measure equivalent to Lebesgue. A diffeomorphism $f: M \rightarrow M$ is ergodic if it is conservative and any $f$-invariant subset of $M$ either has zero measure or full measure. For convenience, we take a non-ergodic diffeomorphism to mean a conservative diffeomorphism which is not ergodic.

A diffeomorphism $f$ on a compact Riemannian manifold $M$ is point-wise partially hyperbolic if there is a $T f$-invariant splitting $T M=E^{s} \oplus E^{c} \oplus E^{u}$ and functions $\sigma, \mu: M \rightarrow \mathbb{R}$ such that $\sigma<1<\mu$ and

$$
\left\|T f v^{s}\right\|<\sigma(p)<\left\|T f v^{c}\right\|<\mu(p)<\left\|T f v^{u}\right\|
$$

for all $p \in M$ and unit vectors $v^{s} \in E_{p}^{s}, v^{c} \in E_{p}^{c}$, and $v^{u} \in E_{p}^{u}$. Further, $f$ is absolutely partially hyperbolic if the functions $\sigma$ and $\mu$ can be taken to be constant.

The distinction between point-wise and absolute partially hyperbolicity is of critical importance when studying systems on the 3 -torus. While there are always unique foliations $W^{u}$ and $W^{s}$ tangent to $E^{u}$ and $E^{s}$, the center bundle $E^{c}$ is not necessarily integrable. A partially hyperbolic diffeomorphism is dynamically coherent if there are invariant foliations tangent to $E^{c} \oplus E^{u}$ and $E^{c} \oplus E^{s}$.

Brin, Burago, and Ivanov proved that every absolutely partially hyperbolic diffeomorphism on the 3-torus is dynamically coherent [2]. Soon after, Rodriguez Hertz, Rodriguez Hertz, and Ures gave an example of a point-wise partially hyperbolic system on $\mathbb{T}^{3}$ which is not dynamically coherent [12]. Further, 
Hammerlindl gave a classification result for absolutely partially hyperbolic systems on the 3-torus [7. As a consequence of the classification, these systems naturally fall into two distinct groups:

- If $f$ is homotopic to Anosov, then every center leaf is dense in $\mathbb{T}^{3}$ and is homeomorphic to a line.

- If $f$ is not homotopic to Anosov, then every center leaf is a circle. These circles form a trivial fiber-bundle over $\mathbb{T}^{2}$ and $f$ can be thought of as a skew-product.

As all known non-ergodic examples fall into the "skew-product" case, this dichotomy provides further motivation for Conjecture 1.11 and Theorem 1.1.

Notation. Throughout this paper, "partial hyperbolicity" is taken to mean point-wise partial hyperbolicity unless the qualifier "absolute" is used. In particular, Theorem 1.1 is proved in the point-wise case.

\section{Outline and Externalities}

The proof of Theorem 1.1 breaks into the following steps. First, using results discovered for three-dimensional, non-accessible systems, we show there is a foliation $W^{u s}$ tangent to $E^{u} \oplus E^{s}$. By the work of Plante, associated to this foliation is a holonomy invariant measure $\mu$, unique up to a constant factor. This measure corresponds to an element of the cohomology $H^{1}\left(\mathbb{T}^{3}, \mathbb{R}\right)$, and as $f$ acts hyperbolically on the cohomology, $f^{*} \mu=\lambda \mu$ for some $\lambda<1$. Then $\mu\left(f^{n} \circ \gamma\right) \rightarrow 0$ as $n \rightarrow \infty$ for any curve $\gamma$ transverse to $W^{\text {us }}$ which implies that $f$ is topologically contracting in the center direction $E^{c}$. From this, we deduce that $f$ is expansive. The work of Vieitez then shows that $f$ is conjugate to Anosov.

In the next section, we assume throughout that $f: \mathbb{T}^{3} \rightarrow \mathbb{T}^{3}$ is a partially hyperbolic system homotopic to Anosov. To avoid confusion, we list in advance the general theorems used.

Given a diffeomorphism $f: M \rightarrow M$, an injectively immersed submanifold $S \subset M$ has Anosov dynamics if $f^{k}(S)=S$ for some non-zero integer $k$ and $\left.f^{k}\right|_{S}$ is Anosov. We say further that $S$ has Anosov dynamics with dense periodic points if $\operatorname{Per}\left(\left.f^{k}\right|_{S}\right)$ is dense in the intrinsic topology of $S$.

Theorem 3.1 (Hertz-Hertz-Ures [11). Let $f: M \rightarrow M$ be a conservative partially hyperbolic diffeomorphism of an orientable 3-manifold $M$. Suppose that the bundles $E^{*}$ are also orientable, $*=s, c, u$, and that $f$ is not accessible. Then one of the following possibilities holds:

1. there is an $f$-periodic incompressible torus tangent to $E^{u} \oplus E^{s}$;

2. there is an $f$-invariant lamination $\varnothing \neq \Gamma(f) \neq M$ tangent to $E^{u} \oplus E^{s}$ that trivially extends to a (not necessarily invariant) foliation without compact leaves of $M$. Moreover, each boundary leaf of $\Gamma(f)$ has Anosov dynamics with dense periodic points; 
3. there is a Reebless invariant foliation tangent to $E^{u} \oplus E^{s}$.

For our problem domain, we show that the third case is the only case possible. Then, we may use the results of Novikov compact leaf theory, as was generalized to the $C^{0}$ case by Solodov [21].

Proposition 3.2. If $\mathcal{F}$ is a Reebless codimension one $C^{0}$ foliation $\mathcal{F}$ of a 3manifold $M$, then

- there is no closed null-homotopic curve transverse to $\mathcal{F}$, and

- for every leaf $L$, the induced map $\pi_{1}(L) \rightarrow \pi_{1}(M)$ is injective.

A key intermediate in proving Theorem 3.1 is the following, which will be used specifically in the next section.

Proposition 3.3 (Hertz-Hertz-Ures [1]). Let $f: M \rightarrow M$ be conservative and partially hyperbolic with one-dimensional center. If $\Lambda$ is a closed, $f$-invariant subset of $M$ consisting of leaves tangent to $E^{u} \oplus E^{s}$, then every component of $\partial \Lambda$ is a leaf having Anosov dynamics with dense periodic points.

These boundary leaves also satisfy the following.

Proposition 3.4 (Franks [5]). If $f: S \rightarrow S$ is an Anosov diffeomorphism and $\operatorname{Per}(f)$ is dense in $S$, then for any periodic point $x \in S$, the unstable manifold $W^{u}(x)$ is dense in $S$.

This is a restatement of (1.7) and (1.8) as given in [5]. Note that the proofs only require $S$ to be connected, not necessarily compact.

The work of Plante shows that many codimension one foliations give rise to holonomy invariant measures. The following is a combination of (4.1) and (7.2) as stated in [16].

Proposition 3.5 (Plante 16]). Let $M$ be a compact manifold such that $\pi_{1}(M)$ has non-exponential growth, and let $\mathcal{F}$ be a codimension one foliation. If $L$ is a leaf which does not intersect any null-homotopic closed transversal, then there is a holonomy invariant measure with support equal to the closure of $L$.

The following is (8.5) from the same paper.

Proposition 3.6 (Plante [16]). Let $\mathcal{F}$ be a codimension one foliation of class $C^{r}(r \geq 0)$ of a compact manifold $M$. If $\mu$ is an $\mathcal{F}$-invariant measure then there is a unique decomposition of $\mu$,

$$
\mu=\mu_{K}+\mu_{1}+\cdots+\mu_{n}
$$

such that the following hold:

1. $\operatorname{supp} \mu_{K}$ is a union of compact leaves.

2. $\operatorname{supp} \mu_{i}$ is connected and is a union of non-compact leaves, $i=1, \cdots, n$. 
3. The sets $\operatorname{supp} \mu_{i}, i=1, \cdots, n$ are pairwise disjoint.

Furthermore, if $\mathcal{F}$ is transversely oriented, $n \leq H_{1}(M ; \mathbb{R}) / 2$.

For a foliation $\mathcal{F}$ of $M$, let a minimal set signify a closed non-empty $\mathcal{F}$ saturated subset which contains no proper subset with the same properties.

Corollary 3.7. If $\mathcal{F}$ is a codimension one foliation without compact leaves on a compact manifold $M$, there are at most a finite number of subsets of the form $X \subset M$ such that $X$ is a minimal set and $X=\operatorname{supp} \mu$ for some holonomy invariant measure $\mu$.

Further, the measures supported on minimal sets are unique up to proportion, as demonstrated in the book of Hector and Hirsch (see Chapter X Theorem 2.3.3 of [8]).

Theorem 3.8 (Hector-Hirsch $[8]$ ). Let $\mathcal{F}$ be a codimension one foliation. Let $\mu$ be $F$-invariant with support a minimal set of $\mathcal{F}$ which is not a compact leaf. If $\mu^{\prime}$ is another $\mathcal{F}$-invariant measure with equal support, there is $c \in \mathbb{R}$ such that $\mu^{\prime}=c \mu$.

Once we establish expansiveness, the final step is to invoke the following result of Vieitez.

Theorem 3.9 (Vieitez [22]). Let $M$ be a three-dimensional compact connected oriented manifold and $f: M \rightarrow M$ an expansive diffeomorphism. If $N W(f)=$ $M$ then $f$ is conjugate to a linear Anosov diffeomorphism and $M=\mathbb{T}^{3}$.

Remark. Here, $N W(f)=M$ is an assumption equivalent to saying that for every non-empty open subset $U \subset M$, there is $k \neq 0$ such that $U$ intersects $f^{k}(U)$. This holds for all conservative diffeomorphisms by Poincaré recurrence. While we assume throughout the next section that $f$ is conservative, we only need this assumption to use that $N W(f)=M$ and to apply Theorem [1.6. In fact, Theorem 3.1 and Proposition 3.3 hold in the non-conservative case, so long as $N W(f)=M$. Therefore, Theorem 1.1 also holds in the non-conservative case if the condition "not ergodic" is replaced by "not accessible and $N W(f)=\mathbb{T}^{3}$."

\section{The proof}

In this section, assume $f: \mathbb{T}^{3} \rightarrow \mathbb{T}^{3}$ is a conservative, non-accessible, partially hyperbolic diffeomorphism homotopic to Anosov. In particular, the action of $f$ on $\pi_{1}\left(\mathbb{T}^{3}\right) \cong \mathbb{Z}^{3}$ is as a hyperbolic linear map. By lifting to a finite cover, assume that the bundles $E^{u}, E^{c}$, and $E^{s}$ are orientable. The original map is expansive if and only if its lift to this finite cover is.

As $f$ is homotopic to Anosov, it forbids certain invariant subsurfaces.

Lemma 4.1. There is no injectively immersed surface $i: S \rightarrow M$ such that $i_{*}: \pi_{1}(S) \rightarrow \pi_{1}(M)$ is injective and $S$ has Anosov dynamics with dense periodic points. 
Proof. Suppose there is such a leaf $S$. As $f^{k}(S)=S$, the image of $\pi_{1}(S)$ in $\pi_{1}\left(\mathbb{T}^{3}\right)$ is an $f_{*}^{k}$-invariant subgroup. As $f_{*}^{k}$ is hyperbolic, the only possibilities for such an invariant subgroup are the trivial group or a full rank subgroup. As no surface has fundamental group isomorphic to $\mathbb{Z}^{3}, S$ must be simply connected. As the 2-sphere does not permit Anosov dynamics, $S$ must be a plane.

By Proposition 3.4 there is a dense unstable leaf through $S$. In the case of a plane, however, if an unstable manifold passes near itself, then by connecting the two nearby segments of the unstable manifold, one can construct a trapping region, a Jordan curve transverse to $E^{u}$ such that the orientation of $E^{u}$ points either entirely in or out of the bound region. This contradicts the fact that the leaf is dense.

Proposition 4.2. There is a unique $f$-invariant foliation tangent to $E^{u} \oplus E^{s}$ without compact leaves.

Proof. We first rule out two of the cases of Theorem 3.1. Suppose there is an $f$-periodic incompressible torus $S$ tangent to $E^{u} \oplus E^{s}$. Then, $\pi_{1}(S) \cong \mathbb{Z}^{2}$ injects into $\pi_{1}\left(\mathbb{T}^{3}\right)$ and the image is invariant under $f_{*}^{k}$ for some $k$. As $f_{*}^{k}$ is hyperbolic, no such rank two subgroup exists, giving a contradiction.

The second case of Theorem 3.1 implies a foliation without compact leaves, some of which have Anosov dynamics with dense periodic points. As the foliation is Reebless, the inclusion of any leaf $\mathcal{L} \subset \mathbb{T}^{3}$ is $\pi_{1}$-injective and Lemma 4.1 gives a contradiction.

Thus, only the third case of Theorem 3.1 is possible, and there is a Reebless foliation tangent to $E^{u} \oplus E^{s}$. As $W^{u}$ and $W^{s}$ are uniquely integrable, the foliation is unique.

Suppose this foliation has a compact leaf $S$ which, as the leaf is foliated by $W^{u}$ and $W^{s}$, must be a 2-torus. Further, as the foliation is Reebless, $\pi_{1}(S)$ injects into $\pi_{1}\left(\mathbb{T}^{3}\right)$. As $f_{*} \pi_{1}(S) \neq \pi_{1}(S)$, we can find a closed loop $\gamma \subset f(S)$ with homotopy class $[\gamma] \in \pi_{1}\left(\mathbb{T}^{3}\right) \backslash \pi_{1}(S)$. By intersection number arguments, $\gamma$ must intersect $S$, but by the unique integrability, we have that $\gamma$ is contained in $S$, a contradiction.

Let $W^{u s}$ denote the foliation tangent to $E^{u} \oplus E^{s}$.

Lemma 4.3. For every minimal set $\Lambda$ of $W^{u s}$, there is a holonomy invariant measure with support equal to $\Lambda$.

Proof. The fundamental group $\pi\left(\mathbb{T}^{3}\right) \cong \mathbb{Z}^{3}$ has non-exponential growth, and as $W^{u s}$ is Reebless, there is no null-homotopic closed transversal by Proposition 3.2. Therefore, if $L$ is a leaf in a minimal set $\Lambda$, by Proposition 3.5, there is a holonomy invariant measure with support equal to $\bar{L}=\Lambda$.

Proposition 4.4. The foliation $W^{u s}$ is minimal; that is, $\mathbb{T}^{3}$ is the only minimal set.

Proof. Suppose $\Lambda$ is a minimal set. As the foliation $W^{u s}$ is $f$-invariant, $f^{k}(\Lambda)$ is also a minimal set for every integer $k$. By Corollary 3.7 and Lemma 4.3, there 
is $k$ such that $f^{k}(\Lambda)=\Lambda$. By Proposition 3.3, using $f^{k}$ in place of $f$, every leaf in $\partial \Lambda$ has Anosov dynamics with dense periodic points, and so by Lemma 4.1. $\partial \Lambda=\varnothing$, which is only possible if the (non-empty) minimal set $\Lambda$ is all of $\mathbb{T}^{3}$.

Now, fix $\mu$ such that $\operatorname{supp} \mu=\mathbb{T}^{3}$. As it has full support, $\mu(\gamma)>0$ for any positive length curve $\gamma$ transverse to $W^{u s}$.

Proposition 4.5. There is $\lambda \neq 1$ such that $f^{*} \mu=\lambda \mu$.

Proof. By Proposition $3.8 \mu$ is unique up to a constant. As the pullback defined by $f^{*} \mu(\gamma)=\mu(f \circ \gamma)$ is another non-zero holonomy-invariant measure, there is $\lambda>0$ such that $f^{*} \mu=\lambda \mu$.

Any path on $\mathbb{T}^{3}$ is homotopic to a path consisting of a concatenation of segments, each either tangent to $W^{u s}$ or transverse. As the foliation is transversely orientable, this canonically associates $\mu$ with a non-zero element $[\mu]$ of the cohomology group $H^{1}\left(\mathbb{T}^{3}, \mathbb{R}\right)$. (See [16] or $[8$ for details.) Further,

$$
\lambda[\mu]=[\lambda \mu]=\left[f^{*} \mu\right]= \pm f^{*}[\mu] .
$$

That is, $\pm \lambda$ is an eigenvalue of the linear map $f^{*}$ on $H^{1}\left(\mathbb{T}^{3}, \mathbb{R}\right) \cong \mathbb{R}^{3}$. As $f$ is homotopic to Anosov, the map $f^{*}$ is hyperbolic, and $\lambda \neq 1$.

By replacing $f$ by $f^{-1}$ if necessary, assume for the remainder of the section that $f^{*} \mu=\lambda \mu$ where $\lambda<1$.

Lemma 4.6. For $\epsilon>0$ there is $\delta>0$ such that for any curve $\gamma$ tangent to $E^{c}$,

$$
\mu(\gamma)<\delta \Rightarrow \operatorname{length}(\gamma)<\epsilon
$$

Proof. Suppose instead that $\left\{\gamma_{k}\right\}$ is a sequence of curves $[0,1] \rightarrow \mathbb{T}^{3}$ such that $\mu\left(\gamma_{k}\right)$ goes to zero, but length $\left(\gamma_{k}\right)$ does not. Take a finite atlas of foliation charts for $W^{u s}$ covering the manifold, and by the Lebesgue Covering Lemma fix $\rho>0$ such that every ball of radius $\rho$ is contained in the domain of one of the charts. Without loss of generality, by replacing each $\gamma_{k}$ by a subcurve, which only decreases its measure, assume length $\gamma_{k}<\rho$ for all $k$. By restricting to a subsequence, further assume that all of the $\gamma_{k}$ lie in the domain $U$ of one of the foliation charts, and that the sequences of endpoints $\left\{\gamma_{k}(0)\right\}$ and $\left\{\gamma_{k}(1)\right\}$ converge.

There is a center curve, $J$, through $U$ such that the $W^{u s}$ holonomy inside the foliation box defines a retract $r: U \rightarrow J$. By transversality of $E^{c}$ to $W^{u s}$, it follows that

$$
\lim _{k} r\left(\gamma_{k}(0)\right) \neq \lim _{k} r\left(\gamma_{k}(1)\right) .
$$

Therefore, $J^{\prime}=\bigcup_{n=1}^{\infty} \bigcap_{k=n}^{\infty} r\left(\gamma_{k}([0,1])\right)$ is a non-trivial subinterval of $J$. Then

$$
\mu\left(J^{\prime}\right) \leq \liminf \mu\left(r\left(\gamma_{k}([0,1])\right)\right)=\liminf \mu\left(\gamma_{k}([0,1])\right)=0 .
$$

This contradicts the fact that $\mu$ has full measure. 
Lemma 4.7. For $\epsilon>0$ there is $\delta>0$ such that for any curve $\gamma$ tangent to $E^{c}$,

$$
\operatorname{length}(\gamma)<\delta \Rightarrow \mu(\gamma)<\epsilon .
$$

The proof is similar to the previous lemma, and is omitted.

Corollary 4.8. For $r, R>0$ there is an integer $N$ such that for any curve $\gamma$ tangent to $E^{c}$ and any $n>N$,

$$
\operatorname{length}(\gamma)<R \Rightarrow \operatorname{length}\left(f^{n} \circ \gamma\right)<r \text {. }
$$

Proof. This is a straightforward consequence of the previous two lemmas, and the fact that $\mu\left(f^{n} \circ \gamma\right)=\lambda^{n} \mu(\gamma)$.

If the center bundle $E^{c}$ were uniquely integrable, it would be easy to prove from the topological contraction in the center direction that $f$ is expansive. However, technical issues arise in trying to prove unique integrability, so we continue the proof without it. First is a form of expansiveness in the $E^{c} \oplus E^{s}$ direction.

Lemma 4.9. There is a constant $\delta>0$ such that the following holds. If $\alpha^{s}, \alpha^{c}$ : $[0,1] \rightarrow \mathbb{T}^{3}$ are paths tangent to $E^{s}$ and $E^{c}$ respectively where $\alpha^{s}(1)=\alpha^{c}(0)$, $x=\alpha^{s}(0)$, and $y=\alpha^{c}(1)$, then $x=y$ if and only if $d\left(f^{n}(x), f^{n}(y)\right)<\delta$ for all $n \in \mathbb{Z}$.

Proof. Fix $r>0$ sufficiently small. By transversality of $E^{s}$ and $E^{c}$, for any $a \in(0,1)$, there is $\delta>0$ such that the following property holds.

If $\gamma^{s}, \gamma^{c}:[0,1] \rightarrow \mathbb{T}^{3}$ are curves tangent to $E^{s}$ and $E^{c}$ respectively, $\gamma^{s}(1)=\gamma^{c}(0)$, and $a r<\max \left\{\right.$ length $\gamma^{s}$, length $\left.\gamma^{c}\right\}<r$ then $d\left(\gamma^{s}(0), \gamma^{c}(1)\right)>\delta$.

Fix $a \in(0,1)$ such that length $(f \circ \gamma)>a$ length $(\gamma)$ for any positive length $C^{1}$ path $\gamma:[0,1] \rightarrow \mathbb{T}^{3}$. This defines $\delta$.

Now suppose $\alpha^{s}, \alpha^{c}$, are as in the statement of the lemma, and that $x=$ $\alpha^{s}(0) \neq \alpha^{c}(1)=y$. We want to find $n \in \mathbb{Z}$ such that $d\left(f^{n}(x), f^{n}(y)\right)>\delta$. If $\alpha^{c}$ or $\alpha^{s}$ has length zero, this task is easy, so assume both are of positive length. Then, $\mu\left(f^{n} \circ \alpha^{c}\right)=\lambda^{n} \mu\left(\alpha^{c}\right)$ implies that length $\left(f^{n} \circ \alpha^{c}\right)$ goes to zero as $n \rightarrow+\infty$ and goes to infinity as $n \rightarrow-\infty$. The definition of partial hyperbolicity implies the same limits for length $\left(f^{n} \circ \alpha^{s}\right)$. Thus, there is $n \in \mathbb{Z}$ such that

$$
\max \left\{\text { length }\left(f^{n} \circ \alpha^{s}\right), \operatorname{length}\left(f^{n} \circ \alpha^{c}\right)\right\}>r
$$

and

$$
\max \left\{\operatorname{length}\left(f^{n+1} \circ \alpha^{s}\right), \text { length }\left(f^{n+1} \circ \alpha^{c}\right)\right\}<r .
$$

The constant $a$ was defined such that

$$
a r<\max \left\{\operatorname{length}\left(f^{n+1} \circ \alpha^{s}\right), \operatorname{length}\left(f^{n+1} \circ \alpha^{c}\right)\right\}<r
$$

which implies that $d\left(f^{n+1}(x), f^{n+1}(y)\right)>\delta$. 
Lemma 4.10. For $\epsilon<0$, there is $\delta<0$ such that if $x, y \in \mathbb{T}^{3}$ satisfy $d(x, y)<\delta$, then there are curves $\gamma^{\sigma}:[0,1] \rightarrow \mathbb{T}^{3}$ for $\sigma=c, s$, u each of length less than $\epsilon$ and such that the concatenation $\gamma^{c} \gamma^{s} \gamma^{u}$ is a path from $x$ to $y$.

Moreover, the point $\gamma^{s}(1)=\gamma^{u}(0)$ is unique. To be precise, if $\alpha^{c} \alpha^{s} \alpha^{u}$ is another such triple of paths, then

$$
\alpha^{s}(1)=\alpha^{u}(0)=\gamma^{s}(1)=\gamma^{u}(0) .
$$

Proof. The existence part of the proof follows from the transversality of the subbundles. Here, we prove the uniqueness claimed above. Suppose $\epsilon>0$ is given. As $W^{u}$ is expanded by $f$, there is $R>0$ such that if $x$ and $y$ are distinct points on the same leaf of $W^{u}$, then

$$
d\left(f^{n}(p), f^{n}(q)\right)>R
$$

for some (possibly negative) integer $n$. For a given integer $N$, there is $r>0$ such that if $d_{u}(p, q)<r$ then $n$ can be taken greater than $N$.

Using Corollary 4.8 fix $N$ such that for any $n>N$ and any curve $\gamma$ tangent either to $E^{c}$ or $E^{s}$

$$
\operatorname{length}(\gamma)<\epsilon \quad \Rightarrow \quad \operatorname{length}\left(f^{n} \circ \gamma\right)<\frac{R}{4}
$$

for all $n>N$. Once $N$ is fixed, fix $r>0$ such that

$$
d_{u}(p, q)<r \quad \Rightarrow \quad d\left(f^{n}(p), f^{n}(q)\right)>R
$$

for some $n>N$.

Now set $\epsilon^{\prime}=\min (r / 2, \epsilon)$ and let $\delta>0$ be the corresponding constant in the existence portion of this lemma. Suppose $x, y \in \mathbb{T}^{3}$ are points such that $d(x, y)<\delta$ and $\gamma^{c} \gamma^{s} \gamma^{u}$ and $\alpha^{c} \alpha^{s} \alpha^{u}$ are triples of paths of length at most $\epsilon^{\prime}$. Let $p=\gamma^{s}(1)=\gamma^{u}(0)$ and $q=\alpha^{s}(1)=\alpha^{u}(0)$. Then, as $p$ and $q$ are connected by the concatenation of four paths of length at most $\epsilon$ tangent either to $E^{s}$ or $E^{c}$, it follows that $d\left(f^{n}(p), f^{n}(q)\right)<R$ for all $n>N$. However, as $p$ and $q$ are also connected by the concatenation of two paths of length at most $r / 2$ tangent to $E^{u}$, if $p \neq q$, it follows that $d\left(f^{n}(p), f^{n}(q)\right)>R$ for some $n>N$. Thus $p$ must equal $q$ and the desired uniqueness is proved.

Proposition 4.11. $f$ is expansive.

Proof. Let $\epsilon_{1}>0$ be small enough that for any two distinct points $p, q$ on the same unstable leaf, there is $n \in \mathbb{Z}$ such that $d\left(f^{n}(p), f^{n}(q)\right)>\epsilon_{1}$. Let $\epsilon_{2}>0$ be small enough that

$$
\text { length } \gamma<\epsilon_{2} \quad \Rightarrow \quad \text { length } f \circ \gamma<\epsilon_{1}
$$

for any $C^{1}$ path in $\mathbb{T}^{3}$. Let $\delta_{1}$ and $\delta_{2}$ be the corresponding constants given by Lemma 4.10. Set $\delta>0$ such that it satisfies the conditions of Lemma 4.9 and is smaller than both $\delta_{1}$ and $\delta_{2}$. 
Now suppose $x_{0}, y_{0} \in M$ are such that $d\left(x_{n}, y_{n}\right)<\delta$ for all $n \in Z$ where $x_{n}=f^{n}\left(x_{0}\right)$ and $y_{n}=f^{n}\left(y_{0}\right)$. By Lemma 4.10, there are paths $\gamma_{n}^{c}, \gamma_{n}^{s}, \gamma_{n}^{u}$ of length at most $\min \left(\epsilon_{1}, \epsilon_{2}\right)$ connecting $x_{n}$ and $y_{n}$. Let $z_{n}$ denote $\gamma_{n}^{s}(1)$. Then as $f \circ \gamma_{n}^{c}, f \circ \gamma_{n}^{s}, f \circ \gamma_{n}^{u}$ are paths of length at most $\epsilon_{1}$ connecting $f\left(x_{n}\right)=x_{n+1}$ to $f\left(y_{n}\right)=y_{n+1}$, it follows by uniqueness, that $f\left(z_{n}\right)=z_{n+1}$ for all $n \in \mathbb{Z}$.

By the choice of $\epsilon_{1}$ at the start of the proof, $z_{n}=y_{n}$ for all $n$, and then by Lemma 4.9 and the choice of $\delta, x_{n}=y_{n}$ for all $n$, proving expansiveness.

\section{$5 \quad$ Higher dimensions}

We now prove a version of Theorem 1.1 in higher dimensions under additional assumptions. For the motivation behind these assumptions, see [7].

Theorem 5.1. Suppose $f: \mathbb{T}^{d} \rightarrow \mathbb{T}^{d}$ is a conservative partially hyperbolic $C^{2}$ diffeomorphism homotopic to a linear Anosov map $A: \mathbb{T}^{d} \rightarrow \mathbb{T}^{d}$. Further, suppose

- $f$ is absolutely partially hyperbolic,

- $f$ has one-dimensional center, and

- the foliations $W^{u}$ and $W^{s}$ of $f$ are quasi-isometric.

If $f$ is not ergodic, it is topologically conjugate to A.

Here, a foliation $W$ on a manifold $M$ is quasi-isometric if, after lifting to the universal cover $\tilde{M}$, there is a constant $Q$ such that $d_{\tilde{W}}(x, y)<Q d_{\tilde{M}}(x, y)+Q$ for all $x$ and $y$ on the same leaf of the lifted foliation $\tilde{W}$.

For the remainder of this section, assume $f$ satisfies the assumptions of Theorem 5.1 and is not ergodic.

Proposition 5.2. No submanifold $S$ tangent to $E^{u} \oplus E^{s}$ has Anosov dynamics with dense periodic points.

Proof. Let $\tilde{S} \subset \mathbb{R}^{d}$ be a connected component of $P^{-1}(S)$ where $P: \mathbb{R}^{d} \rightarrow \mathbb{T}^{d}$ is the universal covering. There is a lift $\tilde{f}: \mathbb{R}^{d} \rightarrow \mathbb{R}^{d}$ of $f$ such that $\tilde{f}^{k}(\tilde{S})=\tilde{S}$. For simplicity, we assume $k=1$.

For $x \in \mathbb{R}^{d}$, consider the set $\bigcup_{y \in W^{u}(x)} W^{s}(y)$. As shown in [7, this set, called the pseudoleaf through $x$, is a complete properly-embedded topological hyperplane in $\mathbb{R}^{d}$, which intersects every center leaf exactly once. If $x$ is in $\tilde{S}$, then the pseudoleaf through $x$ must be contained in $\tilde{S}$, and then, by virtue of completeness, the two submanifolds must coincide. In particular, every center leaf intersects $\tilde{S}$ exactly once.

Let $A: \mathbb{R}^{d} \rightarrow \mathbb{R}^{d}$ be the hyperbolic linear map to which the system is homotopic. That is, the unique linear map $A$ such that $\|A(x)-\tilde{f}(x)\|$ is bounded for all $x \in \mathbb{R}^{d}$. Let $h: \mathbb{R}^{d} \rightarrow \mathbb{R}^{d}$ be a leaf conjugacy from $A$ to $\tilde{f}$ as constructed in 
[7. That is, $A$ may be viewed as partially hyperbolic and $h$ is a homeomorphism such that for any center leaf $\mathcal{L}$ of $A, h(\mathcal{L})$ is a center leaf of $\tilde{f}$ and

$$
\tilde{f} h(\mathcal{L})=h A(\mathcal{L}) .
$$

Further, $h$ satisfies the relation $h(x+z)=h(x)+z$ for all $x \in \mathbb{R}^{d}$ and $z \in \mathbb{Z}^{d}$.

Let $V \subset \mathbb{R}^{d}$ denote the codimension one subspace of $\mathbb{R}^{d}$ spanned by the stable and unstable subspaces of the partially hyperbolic splitting of $A$. Then, $A(V)=V$ and each center leaf of $A$ intersects $V$ in exactly one point.

Suppose $z \in \mathbb{Z}^{d}$ is such that $\tilde{S}=\tilde{S}+z$. Then $x+k z \in \tilde{S}$ for any $x \in \tilde{S}$ and $k \in \mathbb{Z}$. As $\tilde{S}$ is a us-pseudoleaf, [7, Corollary 2.9] implies that if $z \neq 0$ then the sequence

$$
\frac{(x+k z)-x}{\|(x+k z)-x\|}=\frac{z}{\|z\|}
$$

tends to $E_{A}^{s} \oplus E_{A}^{u}$ as $k \rightarrow \infty$. In other words, $z \in V$ and $V=V+z$.

Define $H: V \rightarrow \tilde{S}$ by requiring $H(\mathcal{L} \cap V)=h(\mathcal{L}) \cap \tilde{S}$ for each center leaf $\mathcal{L}$ of $A$. This uniquely determines $H$ and one can verify that it is a true conjugacy, a homeomorphism such that

$$
\tilde{f} H(x)=H A(x)
$$

for all $x \in V$.

Suppose $x \in \tilde{S}$ projects to a periodic point $P(x)=f^{k}(P(x))$. Then, there is $z \in \mathbb{Z}^{d}$ such that $x+z=\tilde{f}^{k}(x) \in \tilde{S}$. Let $y \in V$ be such that $H(y)=x$. Let $\mathcal{L}$ be the center leaf through $y$. Note that

$$
h A^{k}(\mathcal{L})=\tilde{f}^{k} h(\mathcal{L})=h(\mathcal{L})+z=h(\mathcal{L}+z)
$$

and as $h$ is a homeomorphism, $A^{k}(\mathcal{L})=\mathcal{L}+z$. Using that $V=V+z$,

$$
A^{k}(\mathcal{L} \cap V)=A^{k}(\mathcal{L}) \cap A^{k}(V)=(\mathcal{L}+z) \cap(V+z)
$$

and therefore $A^{k}(y)=y+z$.

We have shown that if $x \in \tilde{S}$ projects to an $f$-periodic point on $S$, then $y=H^{-1}(x)$ projects to an $A$-periodic point on $P(V)$. This implies that $A$ has dense periodic points on the invariant submanifold $P(V) \subset \mathbb{T}^{d}$.

The submanifold $P(V)$ can be viewed as $\mathbb{T}^{j} \times \mathbb{R}^{k}$ where $j$ is the rank of $\mathbb{Z}^{d} \cap V$ and $j+k=d-1$. Further, $\left.A\right|_{P(V)}$ can be written as

$$
(x, y) \mapsto\left(A_{1}(x)+B(y), A_{2}(y)\right)
$$

where $A_{1}: \mathbb{T}^{j} \rightarrow \mathbb{T}^{j}$ is a hyperbolic toral automorphism, $A_{2}: \mathbb{R}^{k} \rightarrow \mathbb{R}^{k}$ is a hyperbolic linear map, and $B: \mathbb{R}^{k} \rightarrow \mathbb{T}^{j}$ is linear.

The only way this map can have dense periodic points is if $A_{2}$ is trivial. That is, $k=0$ and $\left.A\right|_{P(V)}=A_{1}$. As both $A$ and $A_{1}$ are linear toral automorphisms, they have determinants equal to \pm 1 when viewed as linear maps. As the eigenvalues of $A_{1}$ consist of all but one of the eigenvalues of $A$, it would imply that the remaining eigenvalue is \pm 1 , contradicting the standing assumption that $A$ is hyperbolic. 
Proposition 5.3. $E^{u} \oplus E^{s}$ is uniquely integrable.

Proof. Let $A C(x)$ denote the accessibility class of $x$, all points reachable from $x$ by taking a concatenation of paths, each tangent to $E^{u}$ or to $E^{s}$. Define

$$
\Gamma(f)=\left\{x \in \mathbb{T}^{d}: A C(x) \text { is not open }\right\} .
$$

One can verify that $\Gamma(f)$ is closed and $f$-invariant, and if $f$ is not accessible then $\Gamma(f) \neq \varnothing$. Somewhat less trivially, $\Gamma(f)$ is laminated by leaves tangent to $E^{u} \oplus E^{s}[10$. By Proposition 3.3, any leaf of $\partial \Gamma(f)$ has Anosov dynamics with dense periodic points, therefore, by the previous proposition, $\partial \Gamma(f)=\varnothing$, and (using that $f$ is not accessible) $\Gamma(f)$ is all of $\mathbb{T}^{d}$.

Proposition 5.4. There is no null-homotopic loop transverse to $E^{u} \oplus E^{s}$.

Proof. Consider the unique foliation $W^{u s}$ tangent to $E^{u} \oplus E^{s}$ on the universal cover $\mathbb{R}^{d}$. As with $\tilde{S}$ in the proof of Proposition [5.2, every leaf of $W^{u s}$ is a complete properly embedded topological hyperplane which intersects every center leaf exactly once. From this, one can see that there is no closed loop on $\mathbb{R}^{d}$ transverse to $W^{u s}$ and therefore no null-homotopic transverse loop on $\mathbb{T}^{d}$.

These last three propositions replace all of the techniques specific to the three-dimensional case that were used in the previous section. Therefore, we may repeat the steps of the previous section to establish a holonomy invariant measure and deduce all of the results of that section, up to and including the fact that $f$ is expansive. The result of Vieitez, however, applies only in dimension three. As such, we must establish the conjugacy directly.

Lemma 5.5. If $\tilde{f}: \mathbb{R}^{d} \rightarrow \mathbb{R}^{d}$ is a lift of $f$ to the universal cover, then for points $x, y \in \mathbb{R}^{d}, x=y$ if and only if the sequence $\left\|\tilde{f}^{n}(x)-\tilde{f}^{n}(y)\right\|$ is bounded for all $n \in \mathbb{Z}$.

Proof. The system has Global Product Structure 7]. As a consequence, for $x, y \in \mathbb{R}^{d}$ there are unique points $p, q \in \mathbb{R}^{d}$ such that $p \in W^{u}(x), q \in W^{c}(p)$ and $y \in W^{s}(q)$.

Suppose $x \neq p$. Then $d_{u}\left(\tilde{f}^{n}(x), \tilde{f}^{n}(p)\right)$ grows exponentially fast as $n \rightarrow \infty$, and, as $W^{u}$ is quasi-isometric by assumption, $\left\|\tilde{f}^{n}(x)-\tilde{f}^{n}(p)\right\|$ grows at the same rate. In particular, by the definition of absolute partial hyperbolicity, this rate of growth is faster than in the stable or center directions, and so $\left\|\tilde{f}^{n}(x)-\tilde{f}^{n}(y)\right\|$ tends to infinity as well.

Hence, we may assume $x=p$ and, by the same logic for the stable direction, that $q=y$. We have reduced to the case where $x$ and $y$ lie on the same center leaf. If $\gamma$ is the center curve connecting these points, then using a holonomy invariant measure $\mu$ as in the previous section, we can show that $\mu\left(\tilde{f}^{n} \circ \gamma\right)$ is unbounded for $n \in \mathbb{Z}$, and hence that $d_{c}\left(\tilde{f}^{n}(x), \tilde{f}^{n}(y)\right)$ is unbounded as well. The center foliation is quasi-isometric [7, from which the result follows.

Proposition 5.6. $f$ is conjugate to Anosov. 
Proof. As $f$ is homotopic to the hyperbolic toral automorphism $A$, there is a semi-conjugacy, a surjective map $h: \mathbb{T}^{d} \rightarrow \mathbb{T}^{d}$ such that $h f(x)=A h(x)$ for all $x \in \mathbb{T}^{d}[5$. We may assume additionally that $h$ is homotopic to the identity, so that $f, h$, and $A$, lift to maps on the universal cover where $\tilde{h} \circ \tilde{f}=A \circ \tilde{h}$ and $\|\tilde{h}(x)-x\|$ is bounded for all $x \in \mathbb{R}^{d}$.

Suppose $\tilde{h}(x)=\tilde{h}(y)$ for points $x, y \in \mathbb{R}^{d}$. By the semi-conjugacy, $\tilde{h} \tilde{f}^{n}(x)=$ $\tilde{h} \tilde{f}^{n}(y)$ and $\left\|\tilde{f}^{n}(x)-\tilde{f}^{n}(y)\right\|$ is bounded for all $n \in \mathbb{Z}$. By the previous lemma, $x=y$. That is, the semiconjugacy $\tilde{h}$ on $\mathbb{R}^{d}$ is injective, and therefore the semiconjugacy $h$ on $\mathbb{T}^{d}$ is injective, making it a true conjugacy between $f$ and A.

\section{$6 \quad$ Full Conjugacy}

Consider a linear automorphism $A: \mathbb{T}^{3} \rightarrow \mathbb{T}^{3}$ with eigenvalues $\lambda_{s}<\lambda_{c}<1<\lambda_{u}$ and the corresponding partially hyperbolic splitting. A small perturbation of $A$ will be both partially hyperbolic and topologically conjugate to $A$. However, the conjugacy may not preserve all of the partially hyperbolic structure. If the perturbation $f$ is accessible, there is no way that the conjugacy can map both $W_{f}^{s}$ and $W_{f}^{u}$ to the corresponding one-dimensional foliations of $A$. In the special case, that $f$ is not accessible, the conjugacy is as good as possible, preserving all of the invariant foliations.

Theorem 6.1. Suppose $f: \mathbb{T}^{3} \rightarrow \mathbb{T}^{3}$ is an absolutely partially hyperbolic diffeomorphism, $E_{f}^{s} \oplus E_{f}^{u}$ is integrable, and $h: \mathbb{T}^{3} \rightarrow \mathbb{T}^{3}$ is a conjugacy from $f$ to a linear hyperbolic toral automorphism $A: \mathbb{T}^{3} \rightarrow \mathbb{T}^{3}$. Then $A$ is partially hyperbolic with a linear splitting such that $W_{A}^{*}=h\left(W_{f}^{*}\right)$ for $*=c, s, u, c s, c u, u s$.

Remark. The following proof generalizes to the case of a $d$-dimensional torus, $d \geq 3$, under the additional assumptions that $W_{f}^{u}$ and $W_{f}^{s}$ are quasi-isometric, and $\operatorname{dim} E_{f}^{c}=1$. As such, we use $\mathbb{T}^{d}$ in place of $\mathbb{T}^{3}$ throughout the proof.

Proof. Assume without loss of generality that $f_{*}=A_{*}$ as automorphisms of $\pi_{1}\left(\mathbb{T}^{d}\right)$, and that $h$ is homotopic to the identity. It follows from Section 2 of [7], that $E_{f}^{c}$ is quasi-isometric and that $f$ and $A$ are absolutely partially hyperbolic and with the same choice of constants in the definition. In particular, there are constants $\hat{\gamma}<1<\gamma$ such that for points $x$ and $y$ on the universal cover,

$$
\begin{aligned}
y & \in W_{f}^{c}(x) \Leftrightarrow \\
C^{-1} \hat{\gamma}^{n} & \leq\left\|f^{n}(x)-f^{n}(y)\right\| \leq C \gamma^{n} \quad \text { for some } C>1 \text { and all } n \in \mathbb{Z} \quad \Leftrightarrow \\
C^{-1} \hat{\gamma}^{n} & \leq\left\|A^{n}(h(x))-A^{n}(h(y))\right\| \leq C \gamma^{n} \quad \text { for some } C>1 \text { and all } n \in \mathbb{Z} \quad \Leftrightarrow \\
h(y) & \in W_{A}^{c}(h(x)),
\end{aligned}
$$

where, by abuse of notation, we use $f, A$, and $h$ to represent the lifted maps on $\mathbb{R}^{d}$. We have shown $W_{A}^{c}=h\left(W_{f}^{c}\right)$.

Assume, without loss of generality, that $A$ has a contracting center direction. That is, $E_{A}^{c} \oplus E_{A}^{s}$ corresponds to the stable direction of the Anosov splitting. 
Then, by considering $\left\|f^{n}(x)-f^{n}(y)\right\|$ as $n \rightarrow \infty$ or $n \rightarrow-\infty$, one shows that $W_{A}^{c s}=h\left(W_{f}^{c s}\right)$ and $W_{A}^{u}=h\left(W_{f}^{u}\right)$. It remains to show $W_{A}^{s}=h\left(W_{f}^{s}\right)$. On the universal cover, fix the leaf $L$ of $W_{A}^{c s}$ passing through the origin. For each $z \in \mathbb{Z}^{d}$, define the map $\tau_{z}: L \rightarrow L, x \mapsto W_{A}^{u}(x+z) \cap L$. Each $\tau_{z}$ is a translation on $L$. The unstable foliation of an Anosov map on a torus $\mathbb{T}^{d}$ is minimal [5]. Therefore, the set of translations $\left\{\tau_{z}: z \in \mathbb{Z}^{d}\right\}$ is dense in the set of all rigid translations of $L$.

As $W_{A}^{u}=h\left(W_{f}^{u}\right)$ and $h\left(W_{f}^{s}\right)$ subfoliate $h\left(W_{f}^{u s}\right)$, one can verify that the translations $\tau_{z}$ preserve the restriction to $L$ of the foliation $h\left(W_{f}^{s}\right)$. By continuity of the foliation, every rigid translation defined on $L$ preserves $h\left(W_{f}^{s}\right)$. Then, a leaf of $h\left(W_{f}^{s}\right)$ is a locally compact, $C^{1}$-homogeneous subset of $L$, and is therefore a $C^{1}$-manifold [20]. The tangent spaces to the leaves are also invariant under every translation of $L$ and therefore the foliation is linear.

Quotienting down to $\mathbb{T}^{d}$, the image of $L$ is dense and so $h\left(W_{f}^{s}\right)$ is linear on all of $\mathbb{T}^{d}$. As a linear invariant foliation, $h\left(W_{f}^{s}\right)$ must equal $W_{A}^{s}$.

\section{The central Lyapunov exponent}

A conservative diffeomorphism is weakly ergodic if almost every point has a dense orbit.

Theorem 7.1. If $f: \mathbb{T}^{3} \rightarrow \mathbb{T}^{3}$ is a conservative partially hyperbolic $C^{2}$ diffeomorphism homotopic to Anosov, then it is weakly ergodic.

Proof. If $f$ is ergodic, it is weakly ergodic. If $f$ is not ergodic, all of the results of Section 4 hold. In particular, each accessibility class is the leaf of a minimal foliation (Propositions 4.2 and 4.4) and is therefore dense. It then follows from the work of Burns, Dolgopyat, and Pesin that almost every orbit is dense [3, Lemma 5].

Theorem 7.2. Suppose $f: \mathbb{T}^{3} \rightarrow \mathbb{T}^{3}$ is a conservative partially hyperbolic $C^{2}$ diffeomorphism homotopic to Anosov. If $f$ is not ergodic, then the central Lyapunov exponent is zero almost everywhere.

Proof. To prove the contrapositive, assume the central Lyapunov exponent is non-zero on a positive measure subset. By the work of Burns, Dolgopyat, and Pesin, there is an ergodic component of $f$ which is open mod zero [3, Theorem 1]. That is, there is $X \subset \mathbb{T}^{3}$ invariant such that $\left.f\right|_{X}$ is ergodic and $X$ differs from a non-empty open set $U \subset \mathbb{T}^{3}$ by a set of measure zero. By Theorem 7.1, the $f$-saturate of $U$ has full measure. Then, $X$ also has full measure which means $f$ is ergodic.

\section{3-normal hyperbolicity}

Suppose $f: \mathbb{T}^{3} \rightarrow \mathbb{T}^{3}$ is an absolutely partially hyperbolic system satisfying the hypotheses of Theorem 6.1. Then, for a center leaf $W^{c}\left(x_{0}\right)$ on the universal 
cover $\mathbb{R}^{3}$ and a deck translation $\tau: \mathbb{R}^{3} \rightarrow \mathbb{R}^{3}$ in $\pi_{1}\left(\mathbb{T}^{3}\right)$, there is a unique map $h_{\tau}: W^{c}\left(x_{0}\right) \rightarrow \tau\left(W^{c}\left(x_{0}\right)\right)$ defined by

$$
h_{\tau}(x) \in \tau\left(W^{c}\left(x_{0}\right)\right) \cap W^{u s}(x) .
$$

Since $W^{c s}\left(x_{0}\right)$ and $W^{c u}\left(\tau\left(x_{0}\right)\right)$ intersect in a unique center leaf, the map $h_{\tau}$ can be viewed as a stable holonomy inside a center-stable leaf followed by an unstable holonomy inside a center-unstable leaf. In the case of a one-dimensional center, both of these holonomies are $C^{1}\left[19\right.$. Therefore, $h_{\tau}$ is $C^{1}$. Further, these maps define a free action of the fundamental group on the center leaf:

$$
\alpha: \pi_{1}\left(\mathbb{T}^{3}\right) \rightarrow \operatorname{Diff}^{1}\left(W^{c}\left(x_{0}\right)\right), \quad \alpha(\tau)=\tau^{-1} \circ h_{\tau} .
$$

Regrettably, a $C^{1}$ action is not enough to prove ergodicity. However, a $C^{2}$ action is.

Lemma 8.1. If $\alpha(\tau)$ (as defined above) is $C^{2}$ for each $\tau \in \pi_{1}\left(\mathbb{T}^{3}\right)$, then $f$ is ergodic.

Proof. We show that $f$ is essentially accessible, that is, every measurable ussaturated subset of $\mathbb{T}^{3}$ has either zero measure or full measure. This is enough to prove ergodicity [4].

Suppose $A \subset \mathbb{T}^{3}$ is such a set and $\tilde{A}$ is its lift to the universal cover. As the $W^{u}$ and $W^{s}$ foliations are absolutely continuous, the intersection $B:=$ $\tilde{A} \cap W^{c}\left(x_{0}\right)$ has zero or full measure if and only if $\tilde{A}$ does. Note that $\alpha(\tau)(B)=B$ for every $\tau$. Take elements $\tau_{1}, \tau_{2} \in \pi_{1}\left(\mathbb{T}^{3}\right)$ such that $\left\langle\tau_{1}, \tau_{2}\right\rangle$ is isomorphic to $\mathbb{Z}^{2}$. Then $C:=W^{c}\left(x_{0}\right) / \alpha\left(\tau_{1}\right)$ is a $C^{2}$ manifold homeomorphic to a circle, and $\alpha\left(\tau_{2}\right)$ defines a $C^{2}$ diffeomorphism of this manifold with an irrational rotation number. In such a case, every measurable, $\alpha\left(\tau_{2}\right)$-invariant subset of $C$ must have either zero Lebesgue measure or full Lebesgue measure [9, Théorème 1.4]. In particular, $B$ has zero measure or full measure and the claim is proved.

To apply this lemma, it is enough to have a point $x_{0}$ such that the manifolds $W^{c s}\left(x_{0}\right)$ and $W^{c u}\left(x_{0}\right)$ are $C^{2}$ and such that the stable/unstable holonomies between center leaves inside these manifolds are $C^{2}$. In fact, if we assume that $W^{c s}\left(x_{0}\right)$ and $W^{c u}\left(x_{0}\right)$ are $C^{3}$, the $C^{2}$ regularity of the holonomies follows.

To see this, first recall the definition of the norm $\|A\|=\sup _{\|v\|=1}\|A v\|$ and co-norm $m(A)=\inf _{\|v\|=1}\|A v\|$ of a linear operator $A: V \rightarrow W$ between normed vector spaces. For a partially hyperbolic system, take $T_{x}^{s} f$ to signify $\left.T_{x} f\right|_{E^{s}(x)}$ and similarly for the superscripts $c$ and $u$.

Theorem 8.2 (Pugh-Shub-Wilkinson [19]). Let $f: M \rightarrow M$ be $a C^{r+1}$ partially hyperbolic diffeomorphism, $r \geq 1$, such that

$$
\left\|T_{x}^{s} f\right\|\left\|T_{x}^{c} f\right\|^{r}<m\left(T_{x}^{c} f\right)
$$

for all $x \in M$. Then, stable holonomies are $C^{r}$ smooth on any $C^{r+1}$ centerstable leaf. 
Corollary 8.3. Let $f$ be a volume-preserving partially hyperbolic $C^{3}$ diffeomorphism on a three-dimensional manifold. Then, stable holonomies are $C^{2}$ smooth on any $C^{3}$ center-stable leaf.

Proof. The Jacobian of $f^{n}$ satisfies

$$
1=J_{f^{n}}(x)=\left\|T_{x}^{s} f^{n}\right\|\left\|T_{x}^{c} f^{n}\right\|\left\|T_{x}^{u} f^{n}\right\| \frac{V(x)}{V\left(f^{n}(x)\right)}
$$

where $V: M \rightarrow \mathbb{R}$ is a continuous, positive function determined by the angles between $E^{s}, E^{c}$, and $E^{u}$. Consequently, there is $C>0$ such that

$$
\left\|T_{x}^{s} f^{n}\right\|\left\|T_{x}^{c} f^{n}\right\|\left\|T_{x}^{u} f^{n}\right\|<C
$$

for all $x$ and $n$. For $n$ sufficiently large, $\left\|T_{x}^{s} f^{n}\right\|\left\|T_{x}^{c} f^{n}\right\| \leq\left\|T_{x}^{u} f^{n}\right\|^{-1} C<1$ and since $E^{c}$ is one-dimensional, $\left\|T_{x}^{c} f^{n}\right\|=m\left(T_{x}^{c} f^{n}\right)$ and Theorem 8.2 is satisfied for $f^{n}$ and $r=2$.

Therefore, to prove ergodicity, we need only find center-stable and centerunstable leaves which are $C^{3}$.

A partially hyperbolic $C^{r}$ diffeomorphism $f: M \rightarrow M$ is $r$-normally hyperbolic if it is dynamically coherent, and

$$
\left\|T_{x}^{s} f\right\|<m\left(T_{x}^{c} f\right)^{r} \leq\left\|T_{x}^{c} f\right\|^{r}<m\left(T_{x}^{u} f\right)
$$

for all $x \in M$.

If $f$ is $r$-normally hyperbolic, its $c s$ - and $c u$-leaves are $C^{r}$ [15, Theorem 6.1].

Theorem 8.4. If $f: \mathbb{T}^{3} \rightarrow \mathbb{T}^{3}$ is volume-preserving, absolutely partially hyperbolic, 3-normally hyperbolic, and homotopic to Anosov, then it is ergodic.

Remark. These last two results need the condition of "volume-preserving" as opposed to just "conservative" as defined in Section 2, This is because the proof of Corollary 8.3 uses that the Jacobian is equal to one.

Remark. In this section, we assumed absolute partial hyperbolicity for convenience. With some work, the above proof can be generalized to point-wise partially hyperbolic systems which are dynamically coherent. Further, R. Potrie has announced that any point-wise partially hyperbolic system $f: \mathbb{T}^{3} \rightarrow \mathbb{T}^{3}$ homotopic to Anosov is dynamically coherent.

Acknowledgements The authors are grateful to Federico and Jana Rodriguez Hertz for many helpful discussions. R.U. was partially supported by ANII grant FCE_2009_1_2687 and A.H. was partially supported by CNPq (Brazil).

\section{References}

[1] A. Avila, J. Bochi, and A. Wilkinson. Nonuniform center bunching and the genericity of ergodicity among $C^{1}$ partially hyperbolic symplectomorphisms. Ann. Sci. de l'Ecole Norm. Sup., 42:931-979, 2009. 
[2] M. Brin, D. Burago, and S. Ivanov. Dynamical coherence of partially hyperbolic diffeomorphisms of the 3-torus. Journal of Modern Dynamics, $3(1): 1-11,2009$.

[3] K. Burns, D. Dolgopyat, and Y. Pesin. Partial hyperbolicity, Lyapunov exponents and stable ergodicity. Journal of statistical physics, 108(5):927$942,2002$.

[4] K. Burns and A. Wilkinson. On the ergodicity of partially hyperbolic systems. Annals of Math., 171(1):451-489, 2010.

[5] J. Franks. Anosov diffeomorphisms. Global Analysis: Proceedings of the Symposia in Pure Mathematics, 14:61-93, 1970.

[6] M. Grayson, C. Pugh, and M. Shub. Stably ergodic diffeomorphisms. Annals of Math., 140(2):295-330, 1994.

[7] A. Hammerlindl. Leaf conjugacies on the torus. PhD thesis, University of Toronto, 2009. To appear in Ergod. Th. and Dyn. Sys.

[8] G. Hector and U. Hirsch. Introduction to the Geometry of Foliations. Part B. Foliations of codimension one. Second edition. Aspects of Mathematics, E3. Friedr. Vieweg and Sohn, Braunschweig., 1987.

[9] M.R. Herman. Sur la conjugaison différentiable des difféomorphismes du cercle à des rotations. Publications Mathematiques de l'IHES, (49):5-233, 1979.

[10] F. Rodriguez Hertz, M. A. Rodriguez Hertz, and R. Ures. Accessibility and stable ergodicity for partially hyperbolic diffeomorphisms with 1D-center bundle. Invent. Math., 172(2):353-381, 2008.

[11] F. Rodriguez Hertz, M. A. Rodriguez Hertz, and R. Ures. Partial hyperbolicity and ergodicity in dimension three. Journal of Modern Dynamics, 2(2):187-208, 2008.

[12] F. Rodriguez Hertz, M. A. Rodriguez Hertz, and R. Ures. A nondynamically coherent example on $\mathbb{T}^{3}$. 2012. preprint.

[13] F. Rodriguez Hertz, M. A. Rodriguez Hertz A. Tahzibi, and R. Ures. New criteria for ergodicity and non-uniform hyperbolicity. Duke Math. J., 160(3):599-629, 2011.

[14] F.R. Hertz, M.A. Hertz, and R. Ures. Tori with hyperbolic dynamics in 3-manifolds. Journal of Modern Dynamics, 5(1):185-202, 2011.

[15] M. Hirsch, C. Pugh, and M. Shub. Invariant Manifolds, volume 583 of Lecture Notes in Mathematics. Springer-Verlag, 1977.

[16] J.F. Plante. Foliations with measure preserving holonomy. Annals of Math., 102(2):327-361, 1975. 
[17] C. Pugh and M. Shub. Stable ergodicity and partial hyperbolicity. In F. et al Ledrappier, editor, 1st International Conference on Dynamical Systems, Montevideo, Uruguay, 1995 - a tribute to Ricardo Mañé, volume 362 of Pitman Res. Notes Math. Ser., pages 182-187. Harlow: Longman, 1996.

[18] C. Pugh and M. Shub. Stable ergodicity and julienne quasi-conformality. Journal of the European Mathematical Society, 2(1):1-52, 2000.

[19] C. Pugh, M. Shub, and A. Wilkinson. Hölder foliations. Duke Math. J., 86(3):517-546, 1997.

[20] D. Repovš, A.B. Skopenkov, and E.V. Šcepin. $C^{1}$-homogeneous compacta in $\mathbb{R}^{n}$ are $C^{1}$-submanifolds of $\mathbb{R}^{n}$. Proc. Amer. Math. Soc, 124(4):12191226, 1996.

[21] VV Solodov. Components of topological foliations. Mathematics of the USSR-Sbornik, 47:329, 1984.

[22] J.L. Vieitez. Lyapunov functions and expansive diffeomorphisms on 3dmanifolds. Ergodic Theory and Dynamical Systems, 22(2):601-632, 2002.

[23] A. Wilkinson. Conservative partially hyperbolic dynamics. In Proceedings of the International Congress of Mathematicians, volume 901, pages 18161836, 2010.

The University of Sydney and the University of New South Wales Mailing address:

Department of Mathematics, University of Sydney, NSW 2006, Australia.

E-mail address: andy@maths.usyd.edu.au

UNIVERSIDAD DE LA REPÚBLICA

Mailing address:

IMERL, Facultad de Ingeniería, CC 30, Montevideo, Uruguay.

E-mail address: ures@fing.edu.uy 